\title{
On soluble just-non-Cross varieties of groups
}

\author{
J. M. Brady
}

\begin{abstract}
We prove a partial confirmation of Kovács and Newman's conjecture that a just-non-Cross variety is soluble if and only if it is in the following list: $\underline{\underline{A}}, \underline{A_{p}} \underline{\underline{A}}, \underline{A_{p}} \underline{\underline{T}}_{q}, \stackrel{A_{p}}{\underline{A}} q_{q} \underline{A}_{r}$, where $p, q$ and $r$ are any three distinct primes.
\end{abstract}

\section{Introduction}

The Oates-Powell. Theorem enables us to call a variety (of groups) Cross if it can be generated by a single finite group. L.G. Kovács and M.F. Newman [7, Theorem 1] have pointed out that a variety is non-Cross if and only if it contains a non-Cross variety whose proper subvarieties are all Cross: a so-called jnC (just-non-Cross) variety. In [6], Kovács and Newman prove that the decomposable (that is, nontrivially factorisable)

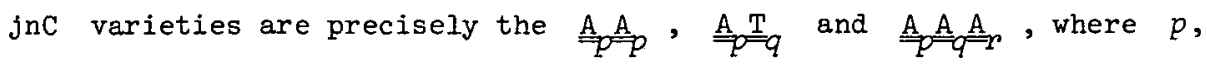
$q$ and $r$ are any three distinct primes, and they propose the following conjecture [6, p. 222].

CONJECTURE 1.1. Every soluble $\mathrm{jnC}$ variety of finite exponent is decomposable.

Our aim in this paper is to substantially reduce Conjecture 1.1 by proving :

Received 24 July 1970. This work was completed while the author was a Ph.D. student holding a Commonwealth Scholarship and Fellowship Plan award at the Australian National University. The author thanks his supervisor, Dr L.G. Kovács, for all the time and effort he has spent on him. He is also indebted to Dr Peter M. Neumann for informing him (after submission of the present paper) that Dr A.Ju. Ol'sanski Y has recently proved Conjecture 1.1. 
THEOREM 1.2. If there is a solubie jnc variety $\underline{\underline{\mathrm{V}}}$ of finite exponent which is not decomposable, then one can find (distinct) primes $p$ and $q$ and an integer $n$ (all three depending on $\underline{V}$ ), such that $\underline{\underline{V}}$ is a subvariety of $\underline{\mathrm{A}}_{p}\left(\underline{\underline{\mathrm{V}}} \wedge \underline{\underline{\mathrm{B}}}_{q}\right)$, where $\underline{\underline{\mathrm{V}}} \wedge \underline{\underline{\mathrm{B}}}_{q} n$ is nilpotent of class at Zeast three.

Theorem 1.2 is phrased so as to emphasise the fact that its hypotheses can be satisfied (if and) only if Conjecture 1.1 is false. Consider the following special case of Conjecture 1.1 .

CONJECTURE 1.3. If $\mathrm{N}$ is a nilpotent variety of class at least two and exponent a power of the prime $q$, and if $p$ is a prime unequal to $q$, then $\stackrel{\mathrm{A} T}{=} q$ is the unique $\mathrm{nnC}$ subvariety of $\stackrel{\mathrm{A}}{\Rightarrow} \underline{\mathrm{N}}$.

COROLLARY 1.4. Conjecture 1.1 is true if and only if Conjecture 1.3 is.

To date, the only significant progress we have made with Conjecture 1.3 is $[2,1.2]$, which shows it to be true in case $\underline{\underline{N}}$ has class two.

COROLLARY 1.5. The soluble $\mathrm{gnC}$ varieties which are not abelian-by-nilpotent are the $\mathrm{A}_{p} \mathrm{~A}_{-}$, where $p, q$ and $r$ are any three distinct primes.

COROLLARY 1.6. The nonmetabelian $\mathrm{jnC}$ varieties which are abelian-by-nilpotent-of-class-two are the $\stackrel{A}{\rightarrow}{ }_{q}$, where $p$ and $q$ are distinct primes.

Theorem 1.2 is a simple consequence of $[1,3.6(i i)],[2,1.2]$, the "internal" result of John Cossey's thesis [3] (which states that A A A is $\mathrm{jnC}$ ), and the following two results.

THEOREM 1.7. If $\underline{\underline{\mathrm{V}}}$ is a soluble $\mathrm{jnC}$ variety which is not locally nilpotent, there are primes $p, q$ and $r$, with $p$ not equal to $q$ or $r$, and a nilpotent subvariety $\underline{N}$ of $\underline{V}$ of exponent a power of $r$, such that $\underline{\underline{\mathrm{V}}} \leq \underline{\underline{\mathrm{A}}} \underline{\underline{\mathrm{A}}} \underline{\underline{N}} \underline{\underline{N}}$ and $p=r$ if and only if $\underline{\underline{\mathrm{V}}}$ is abelian-by-nilpotent.

THEOREM 1.8. Let $p, q$ and $r$ be pairwise distinct primes, and suppose that $\underline{\mathrm{V}}$ is a $\mathrm{jnC}$ subvariety of $\stackrel{\mathrm{A}}{\underline{\mathrm{A}}} \underline{\underline{\mathrm{N}}} \underline{\underline{\mathrm{N}}}$, where $\underline{\underline{\mathrm{N}}}$ is a 
nilpotent variety of exponent a power of $r$. If $\mathrm{V}$ is not

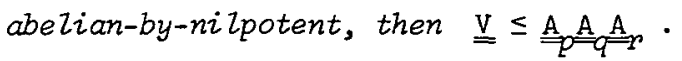

\section{Preliminaries}

Throughout this paper, "group" means "finite group", except in certain places, where its meaning will always be clear from the context. We shall follow as far as possible the notation of Hanna Neumann's book [8], to which we refer for an account of the basic facts about varieties. Notice however that we shall prefer to call a quotient group of a subgroup of a group $G$ a section of $G$. If $G$ is a group, the socle $M(G)$ of $G$ is the product of the minimal normal subgroups of $G ;$ a group is called monolithic if it has precisely one minimal normal subgroup.

We shall often be concerned with restricting a (linear) representation of a group to a normal subgroup, so, rather than refer each time to [4, V, 17.3] (say), we shall make this one reference to it and henceforth feel free to appeal to "Clifford's Theorem". In this direction, we need the following corollary to $[1,2.6]$.

LEMMA 2.1. Let the minimal normal subgroup $M$ of $G$ be abelian of exponent $p$, and consider $M$ as an irreducible $F(p) G$-module ( $G$ acts on $M$ by conjugation), where $F(p)$ is the field of $p$ elements. If $N$ is a normal subgroup of $G$ containing $M$, and $N$ is supplemented in $G$ by a sylow p-subgroup $P$ of $G$, then

(i) the number of homogeneous components of $M_{N}$ is a power of $p$, say $p^{c}$;

(ii) $c$ is less than the class of $P$.

Proof. We shall use Clifford's Theorem. Let $M_{1}, \ldots, M_{k}$ be the homogeneous components of $M_{N}$, and let $H_{1}$ be the inertia group of $M_{1}$. Then $\left|G: H_{1}\right|=k$, and $N \leq H_{1}$. Since $N P=G$, it follows that $\left|G: H_{1}\right|$ is a power of $P$. This proves the first part. The elements of $N$ stabilise each $M_{j}$, and so $P$ must act transitively on $\left\{M_{1}, \ldots, M_{p}\right\}$. Since $P$ contains $M$, it follows from $[1,2.6]$ that 
the class of $P$ is at least $c+1 \cdot / /$

We shall find the following lemma quite useful. We refer to [8, 53.11] for the definition of similarity of normal subgroups.

LEMMA 2.2. Let $\underline{\underline{\mathrm{V}}}$ be a variety, $G$ a group in $\underline{\underline{\mathrm{V}}}$, and $N$ an abelian normal subgroup of $G$. Then $\underline{\underline{V}}$ also contains a group $G^{*}$, which has a complemented, self-centralising (abelian) normal subgroup $N^{*}$, with $N^{*}$ similar to $N$.

Proof. (L.G. Kovács) Denote the direct square $G \times G$ of $G$ by $K$, and the normal subgroup $N \times E$ of $K$ by $M$. Let $L$ be the diagonal in $K$, and denote $L \cap(C \times C)$ by $J$, where $C$ is $C_{G}(N)$. Put $M L / J$ equal to $G^{*}, M J / J$ equal to $N^{*}$, and $L / J$ equal to $H^{*}$. Then $G^{*} \in \underline{\underline{\mathrm{V}}}$, and $N^{*}$ is a self-centralising normal subgroup of $G^{*}$ which is complemented in $G^{*}$ by $H^{*}$. Define $\mu: N \rightarrow N^{*}$ by $n \mu=(n, e) J$, and $\nu: G / C \rightarrow G^{*} / N^{*}$ by $g C \nu=((g, g) J) N^{*}$; it is easy to check that $\mu$ and $v$ are in fact isomorphisms. Finally,

$$
\begin{aligned}
\left(n^{g C}\right)_{\mu} & =n^{g}=\left(n^{g}, e\right) J \\
& =(n, e)^{(g, g)} J \\
& =(n, e) J((g, g) J) N^{*} \\
& ={ }_{n \mu} g C \nu .
\end{aligned}
$$

We conclude this section by recalling Kovács and Newman's version of the Oates-Powell Theorem, as it is the more convenient for our purposes. For positive integers $e, m$ and $c$ denote by $\underline{\underline{\mathrm{c}}}(e, m, c)$ the class of all (not necessarily finite) groups of exponent dividing $e$ whose chief-sections have order (at most) $m$, and whose nilpotent sections have class (at most) $c$. They prove [5]:

THEOREM 2.3. For all positive integers $e, m$ and $c, \underline{\underline{C}}(e, m, c)$ is a Cross variety. Furthermore, a variety $\underline{V}$ is Cross if and only if there exist positive integers $e, m$ and $c$ such that $\underline{\underline{v}}$ is a subclass of $\underline{\underline{\mathrm{c}}}(e, m, c)$. $\quad / /$

\section{The proof of Theorem 1.7}

As $\underline{\underline{V}}$ is not abelian, it has finite exponent, say $n$, and hence it 
is locally finite. Since $\underline{V}$ is not even locally nilpotent by $[1,3.7]$ there is a prime, say $p$, and an infinite set, say $\Gamma$, of monolithic groups in $\underline{\underline{V}}$ such that:

$$
\begin{aligned}
& \text { (i) } \underline{\underline{\mathrm{V}}}=\operatorname{var} \Gamma \text {; } \\
& \text { (ii) the monolith of each group in } \Gamma \text { is complemented, } \\
& \text { self-centralising, and has exponent } p ; \\
& \text { (iii) }\{|M(G)|: G \in \Gamma\} \text { is an infinite set. }
\end{aligned}
$$

We denote by $l$ the least positive integer such that $\underline{\underline{v}}$ is a subvariety of $\underline{\underline{A}}^{Z+l}$; then $\underline{\underline{V}} \wedge \underline{\underline{A}}^{Z}$ is Cross. (Such an $Z$ exists since $\underline{\underline{V}}$ is assumed soluble.) If $\Delta=\left\{G \in \Gamma: G \in \underline{\underline{A}}^{2}\right\}$, then $\operatorname{var} \Delta \leq \underline{\underline{V}} \wedge \underline{\underline{A}}^{2}$, and so it is Cross. It follows that $\operatorname{var}(\Gamma-\Delta)$ is non-Cross, and hence equals $\underline{\underline{V}}$, and so we may as well suppose that $\Delta$ is empty; that is, we suppose that every group in $\Gamma$ has solubility length $l+1$ precisely.

Let $G \in \Gamma$, and denote $M(G)$ by $V$.

LEMMA 3.1. $G^{(\tau)}=V$, and if $k<\tau, G^{(k)}>V$.

Proof. Since $G$ has solubility length precisely $l+1, G^{(k)}$ is trivial if and only if $k \geq 2+1$. Since $G$ is also monolithic, $G^{(k)} \geq V$ for $k \leq l$. As $G^{(2)}$ is abelian, and $V$ is a self-centralising subgroup of $G^{(Z)}, V=G^{(Z)}$. If $k<\tau$, $G^{(k)}>G^{(Z)}=V$. /

Let $H$ be a complement for $V$ in $G$, and observe that by Lemma 3.1, $H \in \underline{\underline{A}}^{2}$. Thus if $\underline{\underline{W}}=\operatorname{var}\{H: G \in \Gamma\}, \underline{\underline{W}}$ is a Cross subvariety of $\underline{\underline{V}}$, and $\underline{\underline{V}}$ is a subvariety of $\stackrel{A}{=} \underline{W}$. By properties (ii) and ( $i i i)$ of $\Gamma$, and $[8,52.24],\{|H|: G \in \Gamma\}$ is an infinite set, and hence there is a positive integer, say $m, 0<m \leq l$, which is least such that $\left\{\left|H^{(m)}\right|: G \in \Gamma\right\}$ is finite, say $\left|H^{(m)}\right|<d$ for all $G \in \Gamma$. From now on we think of $V$ as a faithful irreducible $F(p) H$-module.

LEMA 3.2. $m \geq 2-1$.

Proof. Denote $C_{H}\left(H^{(m)}\right\}$ by $C$; then $\{|H: C|: G \in \Gamma\}$ is 
bounded (by $d !$ ). If $T$ is a (right) transversal of $C$ in $H$, and $W$ is an irreducible submodule of $V_{C}$, it follows from Clifford's Theorem that $V_{C}=\{\{W t: t \in T\}$, and hence that $\{|W|: G \in \Gamma\}$ is infinite. Since $\underline{\underline{V}}$ is $j n C$, we have by Theorem 2.3 that $\underline{\underline{V}}=\operatorname{var}\{V C: G \in \Gamma\}$. But $C^{(m+1)}$ is trivial, and so $\underline{\underline{V}} \leq \underline{\underline{A}}^{m+2}$; that is, $m \geq 2-1$. //

LEMMA 3.3. There is a prime $q$, unequal to $p$, such that either $\underline{V}$ is $\stackrel{\mathrm{A}_{p}}{\mathrm{~T}}=\mathrm{q}$, or $H$ has an abelian normal subgroup $Q$ of exponent $q$ with $\{|Q|: G \in \Gamma\}$ infinite.

Proof. By Clifford's Theorem, $V_{H}\left(z_{-1}\right)$ is completely reducible. Since $V$ is faithful and $H^{(2-1)}$ is abelian, it follows $([4, v, 5.17])$ that $H^{(l-l)}$ is a $p^{\prime}$-group. Thus in case $m=l$ (so that $\left\{\left|H^{(Z-I)}\right|: G \in \Gamma\right\}$ is an infinite set), there is a prime, say $q$, such that $\{|Q|: G \in \Gamma\}$ is infinite, where $Q$ is the socle of the sylow q-subgroup of $H^{(Z-1)}$.

Suppose, on the other hand (using Lemma 3.2), that $m=2-1$. We replace $H$ by $C_{H}\left(H^{(2-1)}\right)$ (as in the proof of Lemma 3.2 ) and apply Lemma 2.2 and Theorem 2.3. In this way, we may suppose that $H^{(2-1)}$ is central in $H$, and in particular that it is cyclic. It follows that $H^{(z-2)}$ is nilpotent of class two, and so, by the argument used for $H^{(2-1)}$ above, it is a $p^{\prime}$-group.

In case $Z=2$, we use Theorem 2.3 to reduce to the case that $H$ is a q-group, for some prime $q$. It then follows from [2, 1.2] that $\underline{v}$ is $\stackrel{A}{=} \stackrel{T}{=}$.

Finally, if $Z>2$, then since $\underline{\underline{v}} \wedge \underline{\underline{A}}^{l}$ is Cross, it follows from Theorem 2.3 that the orders of the irreducible components of $V_{H}(z-2)$ form a finite set as $G$ ranges through $\Gamma$. By Lemma $3.2,\left\{\left|H^{(2-2)}\right|: G \in \Gamma\right\}$ is an infinite set, and so there is a prime, say $q$, such that 
$\{|A|: G \in \Gamma\}$ is infinite, where $A$ is the sylow q-subgroup of $H^{(2-2)}$. Since $H^{(L-1)}$ is cyclic, we have that $A^{(l)}$ is cyclic. Now $V_{A}$ is completely reducible by Maschke's Theorem, and so since $V$ is faithful and $A^{(1)}$ is cyclic, there is an irreducible direct summand, say $U$, of $V_{A}$ such that the kernel $K$ of $U$ avoids $A^{(1)}$. Now $V A \in \underline{\underline{v}}^{\wedge} \underline{\underline{A}}^{Z}$, which is Cross, and so $\{|U|: G \in \Gamma\}$ is a finite set, say $|U|<f$, for all $G \in \Gamma$. Hence $\{|A: K|: G \in \Gamma\}$ is bounded by $f !$. Since $\{|A|: G \in \Gamma\}$ is infinite, it follows that $\{|K|: G \in \Gamma\}$ is infinite. But $K \cap A^{(1)}=E$, and so $K \leq Z(A)$; hence $\{|Z(A)|: G \in \Gamma\}$ is an infinite set, and we can choose $Q=M(Z(A))$. //

If $\underline{\underline{V}}$ is $\stackrel{\mathrm{A}}{\underline{p}} \underline{\underline{\underline{T}}}_{q}$, Theorem 1.7 is proved (with $r=q, \underline{\underline{N}}=\underline{\underline{A}} q$ ), and so we shall assume that $H$ has an abelian normal subgroup $Q$ of exponent $q$ such that $\{|Q|: G \in \Gamma\}$ is infinite. Suppose (using Clifford's Theorem) that $V_{Q}$ has homogeneous components $U_{I}, \ldots, U_{a}(G)$, and denote the kernel of $U_{i}$ by $K_{i}$. Now $V$ is faithful, and so $\bigcap_{i=1}^{a(G)} K_{i}$ is trivial. Since $Q \in \underset{A}{A}, Q / K_{i}$ is (cyclic) of order $q$; hence $\{a(G): G \in \Gamma\}$ is infinite. Now $a(G)$ is the index of the inertia subgroup of $U_{1}$ in $G$, so that every prime dividing $a(G)$ divides $n$. But $n$ is finite, and so there is a prime, say $r$ (which for all we know at this stage could be $p$ ), such that $\left\{a_{p}(G): G \in \Gamma\right\}$ is infinite, where $a_{p}(G)$ is the $r$-share of $a(G)$. If $R$ is a Sylow $r$-subgroup of $G$ and $W$ is an irreducible submodule of $V_{Q R}$, it follows from $[1,2.8]$ that if $W_{Q}$ has $d(G)$ homogeneous components, then $d(G) \geq a_{r}(G)$. In particular, since $\underline{V}$ is $\mathrm{JnC}$, it follows from Theorem 2.3 that $\underline{\underline{V}}=\operatorname{var}\{W Q R: G \in \Gamma\}$, and hence $\underline{\underline{\mathrm{V}}} \leq \underline{\mathrm{A}}_{p} \underline{\mathrm{A}}_{q} \operatorname{var}\{R: G \in \Gamma\}$. Recall that since $\underline{\underline{V}}$ is not locally nilpotent, there is a bound, say $c$, on the nilpotency class of the nilpotent groups in $\underline{\underline{V}}$. Hence $\operatorname{var}\{R: G \in \Gamma\}$ is a nilpotent subvariety $\cong$ of $\underline{V}$ of class at most $c$ and exponent a power of $r$. 
Since $\{d(G): G \in \Gamma\}$ is infinite, there is a group, say $G$, in $\Gamma$ such that $d(G)>p^{c}$. Lemma 2.1 now shows that $r$ is not equal to $p$. Moreover, even $d(G)>I$ implies that $Q$ is not centralized by $R$, so if $q \neq r$ then $Q R$ is not nilpotent and hence neither is $H$. In this case, as $M(G)$ is self-centralizing, $G$ is not abelian-by-nilpotent. Thus if $q \neq r$ then $\underline{\underline{V}}$ is not abelian-by-nilpotent. On the other hand, suppose $q=r$. Then $\underline{\underline{A}} \underline{\underline{N}}$ is locally nilpotent while $\underline{\underline{V}}$ is not, so $\underline{\underline{V}} \wedge \underline{A} q \underline{N}$ is nilpotent; and of course $\underline{\underline{V}} \leq \underline{\underline{A}} \underline{\underline{A}} q \underline{\underline{N}}$ implies $\underline{\underline{V}} \leq \underline{\underline{A}} p(\underline{\underline{V}} \wedge \underline{A} \underline{\underline{N}})$ so now $\underline{\underline{V}}$ is abelian-by-nilpotent. The proof of Theorem 1.7 is complete.

\section{The proof of Theorem 1.8}

Suppose that $\underline{\underline{V}}$ is not abelian-by-nilpotent. Observe that $\underline{\underline{V}}$ is locally finite, being soluble of finite exponent. If $c$ denotes the class of $\underline{\underline{N}}$, then all the nilpotent groups in $\underline{\underline{V}}$ have class at most $c$. It follows that $\underline{\underline{V}}$ is not locally nilpotent, and so by $[1,3.7]$, there is a prime, say $t$, and an infinite set, say $\Gamma$, of monolithic groups in $\underline{\underline{V}}$ such that:

$$
\begin{aligned}
& \text { (i) } \underline{\underline{V}}=\operatorname{var} \Gamma \text {; } \\
& \text { (ii) the monolith of each group in } \Gamma \text { is complemented, } \\
& \text { self-centralising, and has exponent } t ; \\
& \text { (iii) }\{|M(G)|: G \in \Gamma\} \text { is an infinite set. }
\end{aligned}
$$

In every group in. $\underline{\underline{V}}$, the Sylow $p$-subgroup is normal, and its quotient group lies in $\underline{A} \underline{\underline{N}}$. Since $\underline{\underline{V}} \wedge \underline{\underline{A}} q \underline{\underline{N}}$ is Cross, it follows from Theorem 2.3 that

$$
t=p
$$

Suppose $G \in \Gamma$, and denote the monolith $M(G)$ of $G$ by $V$. Let $H$ be a Hall $p^{\prime}$-subgroup of $G$, and let $Q$ be the sylow $q$-subgroup and $R$ a Sylow r-subgroup of $H$. Denote $M(Z(R))$ by $Y$. Then $V$ can be thought of as a vector space over $F(p)$, in which case $H$ is faithfully and irreducibly represented (by conjugation) on $V$. Suppose that $U_{1}, \ldots, U_{a(G)}$ are the homogeneous components of $V_{Q}$, and let $K_{i}$ be the kernel of $U_{i}$. We need to prove three preparatory lemmas. 
LEMMA 4.1. The set $T=\{|Q|: G \in \Gamma\}$ is infinite.

Proof. By property (iii) of $\Gamma,\{|V|: G \in \Gamma\}$ is infinite. As $V$ is a self-centralising chief section of $G$ (property (ii) of $\Gamma$ ), it follows from $[8,52.24]$ that $\{|H|: G \in \Gamma\}$ is infinite. Suppose contrary to the lemma that $T$ is finite, say $|Q|<u$ for all $G$ in $\Gamma$, and denote $C_{H}(Q)$ by $C$. Then $\{|H: C|: G \in \Gamma\}$ is bounded by $u !$, and so by Clifford's Theorem, if $W$ is an irreducible submodule of $V_{C},\{|W|: G \in \Gamma\}$ is infinite. Using Lemma 2.2, we replace $W C$ by $(W C)^{*}$; by Theorem 2.3, $\underline{\underline{\mathrm{v}}}=\operatorname{var}\left\{(W C)^{*}: G \in \Gamma\right\}$. Thus we may suppose that $C=H$, for all $G$ in $\Gamma$. In this case of course $Q \leq Z(H)$, and so $Q$ is cyclic of order at most $q$. It follows that $R$ is normal in $H$ and $|H: R| \leq q$. Then again by Clifford's Theorem, $V_{R}$ is a (homogeneous) direct sum of at most $q$ irreducible submodules of common dimension. Thus there is no bound on the orders of the irreducible submodules of $V_{R}$, as $G$ ranges through $\Gamma$. It follows from Theorem 2.3 that $\underline{\underline{V}}=\operatorname{var}\{V R: G \in \Gamma\}$, and hence that $\underline{\underline{V}}$ is abelian-by-nilpotent, a contradiction. //

COROLLARY 4.2. The set $\Phi=\{|R|: G \in \Gamma\}$ is infinite.

Proof. As $Q / K_{i}$ is faithfully and irreducibly represented on an irreducible component of $U_{i}$, it is cyclic of order $q$. But $V$ is faithful, and so $\cap\left\{K_{i}: 1 \leq i \leq a(G)\right\}$ is trivial. As. $T$ is infinite (Lemma 4.I), it follows that $\{a(G): G \in \Gamma\}$ is infinite. By Clifford's Theorem, $a(G) \leq|H: Q|=|R|$, and so $\Phi$ is infinite. //

LEMMA 4.3. The set $\Psi=\{|Y|: G \in \Gamma\}$ is infinite.

Proof. By Maschke's Theorem, $V_{R}$ is completely reducible; let

$$
V_{R}=V_{1} \oplus \ldots \oplus V_{2(G)}
$$

be a direct decomposition of $V_{R}$ into irreducible submodules. Since $\underline{V} \wedge \stackrel{A}{A}$ is Cross, it follows from Theorem 2.3 that $\left\{\left|V_{i}\right|: I \leq i \leq Z(G), G \in \Gamma\right\}$ is finite, say $\left|V_{i}\right|<m$ for all $i, G$. If the kernel of $V_{i}$ is $R_{i}$, then 


$$
\left\{\left|R: R_{i}\right|: 1 \leq i \leq Z(G), G \in \Gamma\right\}
$$

is bounded by $m !$. Since $V$ is faithful, $\cap\left\{R_{i}: 1 \leq i \leq Z(G)\right\}$ is trivial. Choose a subset, say $\Lambda(G)$, of $\{1, \ldots, Z(G)\}$ which is minimal with respect to $\cap\left\{R_{i}: i \in \Lambda(G)\right\}$ being trivial. Since $\Phi$ is an infinite set (Corollary 4.2), it follows that $\{|\Lambda(G)|: G \in \Gamma\}$ is infinite. We suppose that the components of $V_{R}$ have been numbered so that $\Lambda(G)=\{\lambda, \ldots, k(G)\}$. Put $S_{i}=\cap\left\{R_{j}: 1 \leq j \leq k(G), j \neq i\right\}$, $1 \leq i \leq k(G)$. By the minimality of $\Lambda(G)$, each $S_{i}$ is a nontrivial normal subgroup of $R$, and if $i \neq j, S_{i} \cap S_{j}=E$. Hence

$$
|Y| \geq 1+\sum_{i=1}^{k(G)}\left(\left|S_{i} \cap Y\right|-1\right) .
$$

But for each $i, S_{i} \cap Y$ is nontrivial, so $|Y|>k(G)$. Since $k(G)=|\Lambda(G)|$, and $\{|\Lambda(G)|: G \in \Gamma\}$ is infinite, the proof is complete.

We are now in a position to prove Theorem 1.8. Observe that $Q Y$ is a normal subgroup of $H$ which is supplemented in $H$ by $R$. Thus if $W$ is an irreducible submodule of $V_{Q Y}$, it follows from Clifford's Theorem that

$$
V_{Q Y}=\sum\{W x: x \in R\}
$$

Suppose that $M$ is the kernel of $W$; then the kernel of $W x$ is $M^{x}$. Since $V$ is faithful, it follows that $\cap\left\{M^{x}: x \in R\right\}$ is trivial. But if $x \in R$,

$$
M^{x} \cap Y=(M \cap Y)^{x}=M \cap Y,
$$

and so $M \cap Y$ is trivial. Thus $Y$ acts faithfully on $W$; that is, $Y$ is isomorphic to a subgroup of AutW. In particular, $|W| !>|Y|$. It follows from Lemma 4.3 that $\{|W|: G \in \Gamma\}$ is an infinite set, and so by Theorem 2.3,

$$
\underline{\underline{v}}=\operatorname{var}\{W Q Y: G \in \Gamma\}
$$


Hence $\underline{V}$ is a subvariety of $\stackrel{A}{\Rightarrow} \underset{q}{A}$.

\section{References}

[1] J.M. Brady, On the classification of just-non-Cross varieties of groups", Bull. Austral. Math. Soc. 3 (1970), 293-31l.

[2] J.M. Brady, R.A. Bryce and John Cossey, "On certain abelian-by-nilpotent varieties", BuZZ. Austral. Math. Soc. I (1969), 403-416.

[3] P.J. Cossey, "On varieties of A-groups", Ph.D. thesis, Australian National University, 1966. See also Proc. Internat. Conf. Theory of Groups, Austral. Nat. Univ., Canberra, 1965, 71 (Gordon and Breach, New York, 1967).

[4] Bertram Huppert, Endliche Gruppen I (Die Grundlehren der mathematischen Wissenschaften, Band 134, Springer-Verlag, Berlin, Heidelberg, New York, 1967).

[5] L.G. Kovács and M.F. Newman, "Cross varieties of groups", Proc. Roy. Soc. Ser. A. 292 (1966), 530-536.

[6] L.G. Kovács and M.F. Newman, "Just-non-Cross varieties", Proc. Internat. Conf. Theory of Groups, Austral. Nat. Univ., Canberra, 1965, 221-223 (Gordon and Breach, New York, 1967).

[7] L.G. Kovács and M.F. Newman, "On non-Cross varieties of groups", $J$. Austral. Math. Soc. (to appear).

[8] Hanna Neumann, Varieties of groups (Ergebnisse der Mathematik und ihrer Grenzgebiete, Band 37, Springer-Verlag, Berlin, Heidelberg, New York, 1967).

Australian National University, Canberra, ACT. 